\title{
Opinion \\ The Characteristics of the Interaction of a Magnetic Field with that of the Human Body-Field and Possible Therapeutic Applications
}

Keywords: magnetism, biofield, body-field, electromagnetism, frequency, geomagnetism, therapy, quality, interaction, electricity, geirtz, brugemann, mesmer, hell, bioscalar, steam, motor, phones, TV, radio, adverse affects, CNS, brain, biomagnetism, harmonic

\section{Introduction}

Electromagnetism is 1 of the fundamental Forces in Nature. ${ }^{1}$. You may encounter natural magnetism without electricity but you will never encounter electricity without magnetism. Lodestone is metal magnetized by a lightning strike. ${ }^{2}$ Before the invention of Leyden jars ${ }^{3}$ and other crude batteries, static electricity was the only known form of electricity until Benjamin Franklin. ${ }^{4}$ and others provided evidence that lightning was indeed electrical in characteristic.

With the discovery that a rotating magnetic field produces a current in a wire, the age of electricity was born. ${ }^{5}$. Before electricity was able to power electric motors, steam was the method of choice and used to power the steam engine. Steam is now used to generate electricity in nuclear power plants, nuclear powered submarines and combustible fired power plants. Steam is relatively benign for commercial usage as compared to electrical motors that are very debilitating to the human body-field. Electric motors are well known for debilitating the health of individuals working with them and working in close proximity to them. ${ }^{6,7}$

Today there are 2 major sources of electromagnetic pollution: cell phones and broadcast radio \& television. This article has been written with the intent of proposing a theoretical concept of how a magnetic field interacts with the human body-field, how the quality of the magnetic field follows that of the frequency of the electric component of the electromagnetic field and how the magnetic field may be utilized as a means of therapeutic application.

\section{Discussion}

\section{Interaction}

The earliest recorded use of magnets in the application of a therapeutic mode in Europe was accomplished by Father Maximilian Hell. ${ }^{8}$ Franz Anton Mesmer took the ideas of Hell and ended up proclaiming animal magnetism. ${ }^{9}$ We now know that what Dr. Mesmer called animal magnetism is essentially the intentional projection of bioscalar energy for the purpose of therapy. ${ }^{10,11}$

Every living entity has a biofield that permeates the actual physical body of the entity (plant, animal or microorganism) and projects from the center of the body radially into the environment. The production of the biofield is orchestrated by the genome of the living entity. ${ }^{12}$. The biofield of the human body, or the human body-field, is difficult to characterize with most of the electronic equipment at hand today. ${ }^{13,14}$ There are no wires in the natural physical body and the physical body does produce a detectable magnetic field..$^{15}$ The physical body uses cations and anions ${ }^{16}$ to produce electrical effects. Remember; when there is electricity, there is magnetism. The central nervous system

\author{
Volume I Issue 6 - 2015 \\ Edward F Block \\ Block Institute for Astrobiological Studies, USA
}

Correspondence: Edward F Block, Block Institute For Astrobiological Studies, 375 Dula Springs Road,Weaverville, North Carolina, USA, Email efblockiv@yahoo.com

Received: October 29, 2015 | Published: October 30, 2015

(CNS) of the physical body directly interacts with the electromagnetic radiation of the Solar System as a whole. ${ }^{17}$. The frequency of incident electromagnetic radiation interacts with the CNS to affect behaviors in living systems. ${ }^{18,19}$

The use of a cell phone placed next to the head of a person during a phone conversation is now known to affect the natural biomagnetic field of the human brain. A search online will yield many articles concerning these effects, all adverse! As no electricity passes from the phone to the person, it is the magnetic field of the phone that adversely affects the CNS of the user.

Household electronics and electrical equipment contribute to the electromagnetic pollution in the home. Most usage is temporary, as in the vacuuming of the carpet or the blending of a smoothie. However, radios and TVs are left on for considerable time during usage and thus add considerable abuse to the biofield of a user in close proximity. The physical body resides within the geomagnetic field of the Planet Earth. ${ }^{17,18}$ The water within the body resonates with the Geirtz resonance of $4.55 \mathrm{HZ}^{20}$ that is generated by the interaction of the Birkland currents in the atmosphere with the geomagnetic field. Hans Geirtz shows that the " $m$ " numbers of the equations involved with determining the resonant frequencies of the transverse electromagnetic waves generated by the interaction of the Birkland currents and the geomagnetic field of the Earth have very direct bearing upon the health of all living entities. ${ }^{21}$. In addition, there is a phenomenon known as harmonic generation that occurs with all electromagnetic frequencies where whole number multiples of the main frequency are generated. This is because, even though a particular frequency can be generated as a sine wave as a fundamental frequency, it also nearly always contains other levels of the fundamental frequency at different possible harmonic frequencies. This is the reason for the " $\mathrm{m}$ " numbers, the harmonic frequencies.

\section{Quality}

In order for you to really understand this section, you will need to go to this reference ${ }^{22}$ and read all of the material there slowly and 
very carefully! This is especially true for the 7th and most important Section! Comprehension of the material presented there will go a long way to furthering your education concerning the topic presented herein.

It is the thesis of the author that it is the quality of the magnetic field generated concomitantly with the electrical current that is of therapeutic importance. Cellular components are able to resonate with frequencies from $4.55 \mathrm{~Hz}$ to up to about $10 \mathrm{kHx} .{ }^{23}$ This is well within the range of audible sound and thus the basis of the rationale for using sound ${ }^{24}$ and the accompanying magnetic field as therapy. Indeed, it is only necessary to pass the applied music audio through the speaker coil without the cone to produce a therapeutic benefit, i.e., no actual sound need be applied. ${ }^{25}$

\section{Therapy}

As you might know by now, there is a great deal of basic research and development that needs to be conducted concerning this section. However, you will find many references to producers of therapeutic electronic equipment and espousers of body-field therapy with an online search. This is real and already happening!

As stated above, the $7^{\text {th }}$ Section of the Journal Of Informational Medicine, Issue 2, July 2000 article is important for your understanding of the phenomena that is conceptualized herein. It is the alternating character of effective and disharmonious threapeutic frequency that is crucial. This is reflected in the " $m$ " numbers derived from the equations to determine the resonant, harmonious frequencies of the transverse electromagnetic waves produced by the interaction of the Birkland currents with the geomagnetic field of the Earth. Dr. Geirtz found that, in general, the even " $m$ " numbers were harmonious and the odd "m" numbers were disharmonious to multicellular organisms. The converse was true for microorganisms. Also, harmful chemical substances seemed toresonate at the odd " $m$ " number frequencies. Foods and beneficial herbal substances seemed to resonate at the even "m" number frequencies. Thus, any therapeutic application would need to be done at the even " $m$ " frequencies but further research and development needs to be done to confirm this concept. As an example, the " $\mathrm{m}$ " number for $4.55 \mathrm{~Hz}$ is 6 and the " $\mathrm{m}$ " number for cancer is 4 . Also, the " $m$ " numbers for bacterial infection in humans are 5 and 7. The " $\mathrm{m}$ " number of 66 is $50.16 \mathrm{~Hz}$ which is at the frequency of $50 \mathrm{~Hz}$ AC electrical mains. The " $\mathrm{m}$ " number of 80 is 60.8 which is at the frequency of $60 \mathrm{~Hz} \mathrm{AC}$ electrical mains.

Using the technique pioneered by biophysicist Prof. Cyril W. Smith, Salford University, England, the harmonics of the even "m" frequncies would be the most likely candidates for testing to use as the therapeutic frequencies. A narrow frequency bandpass sweep of the relevant " $m$ " numbers for each patient would need to be done as no 2 people have exactly the same overall organismal body-field. In this manner, a detailed therapeutic mode for each patient visit may be generated and results recorded.

\section{Conclusion}

From the discussion above, the intent of this article to establish a working rational for the usage of the magnetic component of the electromagnetic field for therapeutic application has been met unconditionally. The concepts proposed offer a challenging area for research and development in the fields of physics, biophysics and electrical engineering.

\section{Acknowledgments}

None.

\section{Conflict of interest}

The authors declare that there is no conflict of interest.

\section{Funding}

None.

\section{References}

1. http://www.grandunification.com/hypertext/Fundamental_Forces.html

2. https://en.wikipedia.org/wiki/Lightning

3. https://en.wikipedia.org/wiki/Leyden_jar

4. https://en.wikipedia.org/wiki/Kite_experiment

5. http://inventors.about.com/cs/inventorsalphabet/a/electricity.htm

6. http://www.diamondhead.net/emf.htm

7. http://www.journalinformationalmedicine.org/imf.htm

8. https://en.wikipedia.org/wiki/Maximilian_Hell

9. https://en.wikipedia.org/wiki/Animal_magnetism

10. http://www.diamondhead.net/tmw.htm

11. http://www.journalinformationalmedicine.org/amechbf.htm

12. Block IV EF. The Formation of the Adult Human Body-Chapter 2. Int $J$ Complement Alt Med. 2015;1(3):00015.

13. http://www.journalinformationalmedicine.org/hbf.htm

14. http://www.diamondhead.net/p13.htm

15. https://en.wikipedia.org/wiki/Biomagnetism

16. Edmonds DT. Electricity and Magnetism in Biological Systems. Hyderabad, India: Oxford University Press; 2001. pp. 286.

17. http://www.journalinformationalmedicine.org/cr1.htm

18. http://www.journalinformationalmedicine.org/cr2.htm

19. http://www.diamondhead.net/p14.htm

20. http://journalinformationalmedicine.org/bigs.htm

21. http://www.miklagaard.com/papers/paper-1b-3/

22. http://www.diamondhead.net/p2-i.htm

23. http://www.diamondhead.net/p2-5.htm

24. Imprinted Music.

25. Goldman J. Healing Sounds. 